\title{
Estudio de LIDERAZGo Educativo EN UN Modelo de Gestión Escolar hacia la Calidad
}

\author{
Study of Educational Leadership in a Quality \\ Based School Management Model
}

Pablo López Alfaro

Especialista en Gestión y Liderazgo Escolar Departamento de Educación, Universidad de Chile Capitán Ignacio Carrera Pinto 1045, Ñuña, Santiago pablopez@uchile.cl

Resumen: El presente trabajo aborda la importancia del liderazgo en las comunidades escolares en Chile y su relación con la calidad educativa. Se postula un modelo causal que recoge variables relevantes asociadas a la gestión escolar, entre las que se encuentra la variable liderazgo.

El estudio pone de manifiesto esta realidad en torno a las escuelas como organizaciones. A medida que pasa el tiempo, éstas se transforman en sistemas cada vez más complejos, siendo el liderazgo que se ejerce en un centro educativo, una de las variables fundamentales que influye en el logro de los objetivos propuestos por la organización y que permite a sus actores enfrentar exitosamente su complejidad, de acuerdo a lo señalado en diversos estudios nacionales e internacionales.

Palabras clave: Liderazgo, gestión escolar, calidad educativa, modelo causal.

Abstract: This paper deals with the importance of leadership in school communities in Chile and its relationship to educational quality. A causal model is proposed that incorporates relevant variables associated with school management, among them leadership.

The study highlights this reality about schools as organizations. With the passing of time, they become increasingly complex systems, where the leadership exercised in a school is one of the key variables that influences the achievement of the organization's objectives and allows its actors to successfully face its complexity, as noted in several national and international studies.

Keywords: Leadership, school management, educational quality, causal model. 


\section{INTRODUCCIÓN}

A medida que el país alcanza un mejor estado de desarrollo, las organizaciones no educacionales han incorporado paulatinamente a su quehacer, elementos de liderazgo, planificación estratégica, desarrollo organizacional y evaluaciones de la calidad de su gestión, aplicando planes sistemáticos de mejoramiento. Este acento en la gestión también se ha manifestado en el ámbito de las instituciones educativas.

Por otro lado diversos estudios nacionales e internacionales han demostrado la importancia de la gestión escolar, y del liderazgo en particular, en los resultados de aprendizaje de los alumnos y en la calidad educativa de los centros. El liderazgo escolar se ha constituido en una prioridad dentro de la política pública diseñada desde el Ministerio de Educación (2005), lo que se ha traducido en potenciar el liderazgo en el sistema educativo, para impulsar el trabajo en equipo y la generación de ambientes de trabajo profesional que permitan avanzar en materia de calidad y equidad de la educación. Asimismo, la Fundación Chile (2003) en colaboración con la Pontificia Universidad Católica, instalan un modelo denominado procesual para evaluar y certificar la calidad de la gestión escolar, donde la variable liderazgo es uno de los componentes principales en la explicación de los procesos de calidad.

Para Coronel (2008), el liderazgo es un tema que sigue recibiendo una atención destacada y abundante en trabajos empíricos, reflexiones académicas y publicaciones. No se puede discutir ni poner en duda que estamos frente a un fenómeno real y de gran importancia para el análisis organizativo y el funcionamiento de la vida social. En las organizaciones educativas hablamos de liderazgo educativo que nos hace distinguirnos, de otros acercamientos y abordajes.

Maureira (2008) sostiene que el estudio del liderazgo aporta un sinnúmero de enfoques y reflexiones en torno a este fenómeno, haciéndose notar como un tema ampliamente estudiado pero, según algunos autores, el peor comprendido. Parece clara la complejidad del tema a nivel de la organización escolar, en donde el énfasis del trabajo se fundamenta esencialmente en la interacción de distintos agentes educativos: alumnos, profesores, directivos docentes y otros. 


\section{GESTIÓN Y CALIDAD}

El concepto de calidad está presente en todos los ámbitos de la vida. Aparece asociado a aquello que queremos destacar como atractivo o valioso, a la excelencia, al trabajo bien hecho o a aquello que se quiere reconocer. Marchesi y Martin la conciben como, "Un anhelo, un deseo de perfección, un objetivo al cual aproximarse pero que nunca se consigue del todo" (1998, p. 10). Ambas definiciones parecen subrayar los elementos objetivos del concepto, olvidando que éste tiene además un elemento subjetivo: se fundamenta en las necesidades del beneficiario, por lo que los parámetros para medir calidad no están asociados sólo con las características intrínsecas de un producto o servicio.

El concepto ha evolucionado desde un enfoque centrado en las características objetivas del producto o servicio a un enfoque que incorpora elementos de subjetividad, lo cual hace al concepto de calidad alejarse de la consideración del costo como su fundamento y lo convierte en un concepto dinámico, llegando en la actualidad a referirnos permanentemente a gestión de calidad o gestión bien hecha.

La gestión de calidad se basa en un modelo que hace uso intensivo de las personas y no en el capital físico o financiero de una organización, de tal manera que interesa por sobremanera la calidad de las personas que pertenecen a la organización, su integridad, así como la de quienes lideran los procesos. Esta perspectiva obliga a enfatizar la necesidad de las organizaciones no sólo de lanzar un buen producto al exterior, sino además funcionar bien internamente, es decir no es posible que una organización orientada hacia el beneficiario no esté orientada hacia las personas. Si se logra un buen funcionamiento interno y que los trabajadores se sientan bien tratados y a gusto en la organización, éstos querrán agradar a los beneficiarios. Albrecht señala que "Los empleados descontentos son terroristas. Se lo propongan o no, destruyen la calidad del servicio desde la base" (1995, p. 90).

El concepto de calidad ha evolucionado, desde una visión tradicional como plantean Gaziel et al (2000), a una visión transformadora. En la primera la organización actuaba sobre los efectos; con un control de calidad a posteriori, el campo de acción estaba limitado a la calidad del producto y se pensaba que era normal que algunos productos fuesen defectuosos, porque la calidad era costosa. La organización estaba centrada en los problemas, no en los beneficiarios a quienes se les intentaba justificar los defectos en el servicio o producto. 
La visión transformadora de la calidad, que la acerca al concepto de gestión de calidad, se centra en un control a priori para evitar o prevenir defectos. Su campo de acción es toda la organización y su gestión está basada en una investigación permanente del usuario, lo que lleva a la organización a estar centrada en la satisfacción del beneficiario, en conocer sus necesidades. La gestión de calidad se basa en principios que incluyen su consideración no como un problema aislado, sino abarcando toda la organización y orientando la gestión hacia el usuario externo, el cual es estimado como lo más importante, sin dejar de lado al beneficiario interno cuyo bienestar es determinante en los resultados de la gestión de calidad.

Bajo esta conceptualización, la calidad está modelando la gestión organizacional en todos sus ámbitos constituyendo un marco regulatorio del quehacer institucional que al igual que los conceptos de liderazgo, planificación y visión imprimen direccionalidad a la gestión, enmarcan la acción de los componentes de la organización y constituyen partes de un engranaje donde la gestión juega un rol articulador de estos elementos que moviliza a la organización hacia el cumplimiento de su propósito.

\section{GESTIÓN Y LIDERAZGO}

Lepeley concibe el liderazgo como, "La ciencia de conducir a una organización de la situación actual a una situación futura comparativamente mejor, donde las personas son el principal factor de cambio", noción que se relaciona ampliamente con el concepto de gestión, no obstante la diferencia entre ambos conceptos (2001, p. 23).

El liderazgo, como hecho social y como concepto científico, sigue navegando en un mar de imprecisiones, no sólo por la amplitud de su contenido, sino también por la variedad de perspectivas desde donde se le ha enfocado, lo que lo ha llevado a evolucionar desde un enfoque netamente psicológico hasta una perspectiva de interacción social y a mantener latente su relación con autoridad y poder, llevando incluso a generar imprecisiones entre los tres conceptos.

Ruiz entiende por liderazgo, "El arte de conseguir que las personas cumplan una tarea voluntariamente", definición que, asociada a lo planteado por el mismo autor -que "El liderazgo equivale al ejercicio de la autoridad que consigue una obediencia entusiasta de parte de sus súbditos"- nos lleva a reflexionar respecto del sentido que el liderazgo tiene para una organización (1995, p. 371). 
Difícilmente una organización puede desarrollar una buena gestión si no mantiene un liderazgo efectivo que la haga trascender desde un plano operativo, del día a día, a uno de más amplio plazo relacionado con su desarrollo. Resulta esencial establecer que mientras el liderazgo está vinculado estrechamente al desarrollo de una visión y estrategias, la gestión se encuentra asociada a la mantención del funcionamiento de un sistema, relacionándose más bien con la efectividad de la realización de las tareas que con la dirección, sostenida por una estructura organizativa y con funciones administrativas como la planificación, organización y control.

Para algunos autores, como López Ruperez, el modelo en esta dimensión incluye la consideración de cómo el liderazgo del equipo directivo inspira y conduce hacia la calidad total, dentro de un proceso que se considera fundamental para la organización y que se entiende como de mejora continua. El liderazgo del equipo directivo es conceptualizado como, "El compromiso efectivo de los cuadros directivos de la organización y del director general con la calidad, que en la práctica significa la definición de los objetivos de calidad por parte del máximo nivel ejecutivo y orientar a toda la organización en ese sentido" (1997, p. 48).

Gaziel et a l. consideran al Liderazgo como un criterio a evaluar, un agente facilitador del proceso y lo definen como, "...el comportamiento y actuación del Equipo Directivo y del resto de los responsables para guiar a la organización hacia la calidad. A través de él, se pretende evaluar cómo aquellos que tienen alguna responsabilidad en la organización estimulan, apoyan y fomentan la gestión de calidad, como un proceso de mejora continua" (2000, p. 188).

Una de las características más relevantes del liderazgo, desde la perspectiva de una gestión de calidad, es la capacidad de generar ambientes al interior de la organización que alienten la innovación, de tal manera que todos sus miembros se sientan motivados a aportar ideas para el mejoramiento continuo en todos los ámbitos de la gestión.

En los últimos años en la realidad chilena, también se han venido incorporando los aportes de modelos de gestión de calidad. Uno de ellos es el desarrollado por el Ministerio de Educación, denominado SACGE (Sistema de Aseguramiento de la Calidad de la Gestión Escolar) conformado por cinco elementos de gestión: liderazgo, gestión curricular, convivencia escolar, recursos y resultados.

Para la elaboración del modelo teórico a partir del cual se diseñaron los instrumentos de recolección de información, se consideraron dos trabajos. El 
primero corresponde al Modelo Europeo de Calidad EFQM(1998) y el segundo correspondió al realizado por el MINEDUC (2005) en el desarrollo del modelo SACGE. A partir de de estos trabajos, en esta investigación se propone un modelo teórico que da cuenta de siete dimensiones que explican la calidad de los centros educativos y que corresponden a: i) Liderazgo escolar; ii) Planificación y estrategia; iii) Gestión de recursos; iv) Gestión de personas; v) Procesos; vi) Satisfacción y vii) Resultados.

\section{METODOLOGÍA DEL ESTUDIO}

Este estudio presenta la evaluación del liderazgo educativo en la gestión escolar de setenta establecimientos educativos de Enseñanza Básica y Media por la relevancia que tiene en la calidad educativa. Se realizaron análisis factoriales, exploratorios, y análisis causal utilizando ecuaciones estructurales. El modelado con ecuaciones estructurales incluye dos submodelos (Cea, 2002):

i) El modelo estructural: nos permitió describir las relaciones causales entre variables latentes mediante ecuaciones estructurales lineales, que expresan la estructura causal asumida entre las variables.

ii) En el modelo de medición se establecieron las relaciones de las variables latentes con sus indicadores.

De acuerdo con datos empíricos y consideraciones teóricas, planteamos el siguiente modelo (proceso - producto) como consecuencia de la interacción de los comportamientos docentes y de estudiantes.

Tabla 1. Modelo proceso-producto, el liderazgo como componente del modelo gestión hacia la calidad educativa

\begin{tabular}{|c|c|}
\hline Proceso & \\
\hline Liderazgo educativo & \\
\hline Planificación y estrategia & \\
\hline Gestión de recursos & Producto \\
\hline Procesos & Calidad educativa \\
\hline Gestión de personas & \\
\hline Satisfacción & \\
\hline Resultados & \\
\hline
\end{tabular}


Las variables de gestión escolar se recogieron a través del cuestionario diseñado para la encuesta, cuya validación empírica se realizó utilizando ecuaciones estructurales con variables latentes; las que fueron modeladas utilizando el software LSREL (Linear Structural Relations). (Jöreskog y Sörbom, 1993).

Las dimensiones y variables del instrumento se muestran en la tabla $\mathrm{N}^{\mathrm{o}} 2$. Los descriptores de cada variable fueron extraídos desde el modelo Europeo de Calidad EFQM (1998), y del modelo SACGE (2005) mencionadas anteriormente.

Tabla 2. Dimensión liderazgo y otras variables asociadas a la gestión escolar

\begin{tabular}{|c|c|c|l|}
\hline Dimensiones & \multicolumn{1}{|c|}{ Variables } & Dimensiones & \multicolumn{1}{|c|}{ Variables } \\
\hline Liderazgo & $\begin{array}{c}\text { Compromiso } \\
\text { reconocimiento }\end{array}$ & Satisfacción & $\begin{array}{l}\text { Nivel de agrado con la } \\
\text { gestión y funcionamiento } \\
\text { del establecimiento }\end{array}$ \\
\hline $\begin{array}{c}\text { Planificación y } \\
\text { estrategia }\end{array}$ & $\begin{array}{c}\text { Desarrollo de la estrategia } \\
\text { Comunicación }\end{array}$ & Resultados & $\begin{array}{l}\text { Logros del } \\
\text { establecimiento en todos } \\
\text { sus ámbitos de gestión }\end{array}$ \\
\hline $\begin{array}{c}\text { Gestión de } \\
\text { personas }\end{array}$ & Competencias \\
Recursos & $\begin{array}{l}\text { Gestión de recursos } \\
\text { Innovación }\end{array}$ & $\begin{array}{l}\text { Expectativas } \\
\text { Percepciones de los } \\
\text { docentes en los ámbitos } \\
\text { de gestión }\end{array}$ \\
\hline Procesos & $\begin{array}{l}\text { Identificación de procesos } \\
\text { Seguimiento y control de } \\
\text { procesos }\end{array}$ & & \\
\hline
\end{tabular}

La muestra del estudio estuvo compuesta por 70 establecimientos de las regiones V, VIII y Metropolitana: 32 (45,7\%) municipales, 23 (32,8\%) particulares subvencionados y $15(21,4 \%)$ particulares pagados. Los instrumentos (Escala tipo Likert) desarrollados y validados fueron aplicados a una muestra de 870 profesores y directivos trabajando con una base de 60.000 datos.

La primera tarea consistió en desarrollar los análisis de fiabilidad inicial y unidimensionalidad de cada una de las subescalas.

En la segunda etapa se realizaron los análisis factoriales confirmatorios de ambos modelos de medición, tanto de variables latentes exógenas (independientes) como endógenas (dependientes) definidas en el modelo. 
En la tercera etapa se aplicaron los sistemas de ecuaciones estructurales en la validación del modelo causal, en conjunto con el modelo estructural y de medición.

\section{RESULTADOS}

Los resultados que se presentan a continuación, corresponden a análisis que se focalizan en aquellos datos que acentúan la gestión escolar y las variables asociadas destacando la dimensión liderazgo escolar y su relación con la calidad educativa en los establecimientos primarios y secundarios que se incluyeron en el estudio.

\section{Estudio 1.}

i) Análisis de la fiabilidad del Instrumento. Los cálculos de los coeficientes de confiabilidad de las dimensiones originales del instrumento adoptada de los modelos SACGE y EFQM y conformada por las variables: Liderazgo, Planificación, Gestión de Recursos, Personas, Procesos, Satisfacción, Resultados y Calidad Educativa, nos indicaron que la escala general muestra niveles de consistencia interna $\left({ }_{-}=0.96\right)$. De manera similar constatamos una alta consistencia interna en la medida para cada una de las variables asociadas con la gestión escolar.

Tabla 3. Valores de la fiabilidad referida a las variables asociadas a la gestión escolar

\begin{tabular}{|c|c|c|}
\hline Dimensión teórica & Valor "a" & Número de ítems \\
\hline Liderazgo & 0.88 & 9 \\
\hline Planificación y Estrategia & 0.88 & 7 \\
\hline Personas & 0.87 & 11 \\
\hline Recursos & 0.77 & 5 \\
\hline Procesos & 0.85 & 8 \\
\hline Satisfacción & 0.90 & 7 \\
\hline Resultados & 0.84 & 4 \\
\hline Calidad Educativa & 0.72 & 60 \\
\hline Variables asociadas a la Gestión Escolar & 0.96 & \\
\hline
\end{tabular}

ii) Análisis factorial explora torio (AFE) para va riables la tentes exógenas o independientes. Esta exploración permitió determinar en qué medida, la agrupación de indicadores se corresponde con los planteamientos teóricos, para luego desarrollar análisis posteriores con fines confirmatorios. (Hair et al., 2001). 
En primer lugar la matriz de correlaciones presenta valores que superan 0,30 en valor absoluto, además todas las correlaciones tienen un grado de significación inferior a 0.001 . Este dato nos señala la conveniencia de proseguir con la factorización.

El valor de adecuación muestral (KMO) presenta un indicador de 0,916, lo cual ratificó la decisión de agrupar variables en factores comunes. La prueba de Bartlett (sig $=0.00)$ presenta una valor que permite determinar que las correlaciones observadas de la muestra están correlacionadas con la población de las que han sido extraídas.

Con respecto al método de extracción de factores de componentes principales, se observó la presencia de cinco factores con un valor superior a la unidad que en conjunto explican el $58 \%$ de la varianza de los datos.

La conformación de los factores que mostró la matriz de componentes rotados, dada las variables que lo saturan, son los siguientes.

Tabla 4. Matriz de componentes rotados

\begin{tabular}{|l|l|l|l|l|l|}
\hline \multirow{2}{*}{} & \multicolumn{5}{|c|}{ Componente } \\
\cline { 2 - 6 } & \multicolumn{1}{|c|}{1} & 2 & 3 & 4 & 5 \\
\hline Lider 5 & .625 & & & & \\
Lider 7 & .616 & & & & \\
Lider 9 & .743 & .565 & & & \\
Plani 11 & & .568 & .802 & & \\
Plani 15 & & & .836 & & \\
Perso 18 & & & .781 & \\
Perso 19 & & & & .789 & .828 \\
Recur 28 & & & & & .775 \\
Recur 31 & & & & & \\
Proce 37 & & & & & \\
Proce 38 & & & & & \\
\hline
\end{tabular}

iii) Análisis factorial exploratorio, variables latentes endógenas o dependientes. En este estudio la matriz de correlaciones presentó valores satisfactorios, de la misma forma que los índices tanto de adecuación muestral $(\mathrm{KMO}=0.944)$ como del test de Bartlett (Sig. 0.00). En relación a la extracción de componentes principales, se observó la presencia de tres factores que, en conjunto, explican el $60 \%$ de la varianza.

La conformación de los factores que mostró la matriz de componentes rotados, es la siguiente. 
Tabla 5. Matriz de componentes rotados

\begin{tabular}{|l|l|l|l|}
\hline \multirow{2}{*}{} & \multicolumn{3}{|c|}{ Componente } \\
\cline { 2 - 4 } & \multicolumn{1}{|c|}{1} & 2 & 3 \\
\hline Satis45 & .735 & & \\
Satis48 & .816 & & \\
Satis49 & .849 & & \\
Resul45 & & .666 & \\
Resul55 & & .676 & \\
Resul56 & & .448 & \\
Calid57 & & & .896 \\
Calid59 & & & .670 \\
\hline
\end{tabular}

Finalmente, es importante consignar que el análisis factorial exploratorio proporciona sólo un criterio matemático, orientándonos hacia la validación de los modelos de medida para las distintas variables que forman parte de nuestro estudio.

\section{Estudio 2.}

Estimación del modelo de relaciones con ecuaciones estructurales.

A partir de un conjunto de análisis realizados con el objetivo de encontrar el mejor modelo que se ajustará a los datos, encontramos coeficientes estimados en los modelos de medida y estructural que excedían los límites aceptables. Por ejemplo, varianza de error negativa, varianzas de error no significativa para los constructos y coeficientes estandarizados que sobrepasaban el valor aceptable de 1.0, esto significó eliminar los indicadores plani11, plani15, perso19, satis48, resul54 y resul56.

Modelo final. Diagrama del modelo final y sus correspondientes índices de bondad de ajuste. 


\section{Figura 1. Modelo Final}

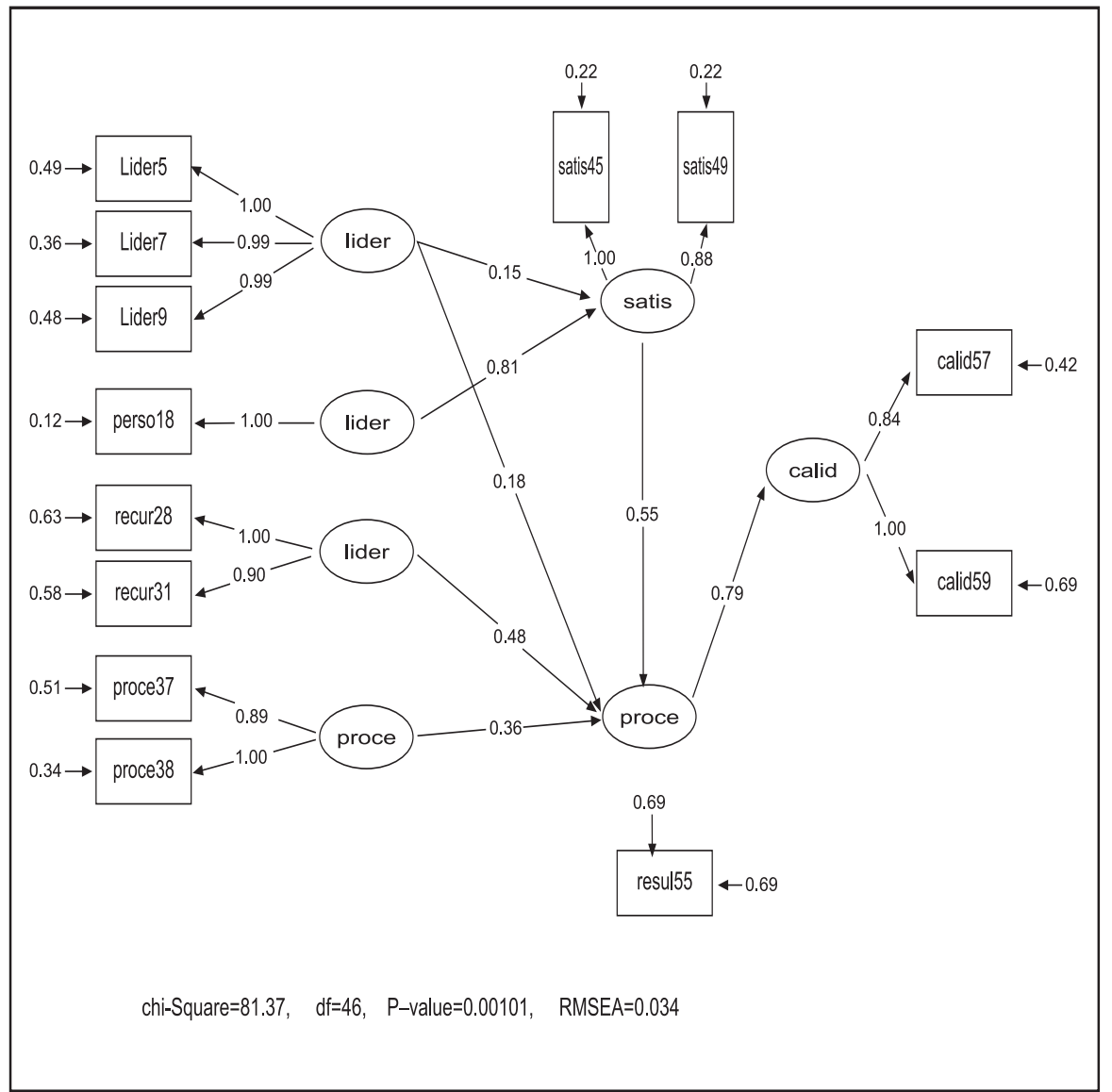

El modelo final, presenta saturaciones significativas distintas de cero, las estimaciones de varianzas de error y varianzas de perturbación, son estadísticamente significativas.

Índices de bondad de ajuste del modelo final para el diagnóstico global. Este análisis, permitió descubrir el grado de ajuste entre el modelo y los datos.

Tabla 6. Índices de ajuste para el modelo final

\begin{tabular}{|c|c|c|c|c|}
\hline CFI & NFI & GFI & RMSEA & $\chi^{2}$ Normado \\
\hline .995 & .993 & .978 & 0.27 & 1.91 \\
\hline
\end{tabular}


El ajuste es positivo en todos los índices obtenidos para el modelo que agrupa en siete variables latentes los trece indicadores. En este sentido, los índices CFI, NFI, GFI, $\chi^{2}$ Normado y RMSEA, todos muestran valores considerados muy buenos. Finalmente se realizó un análisis al modelo final con diferentes submuestras, tanto por separado como a través del análisis multimuestra.

\section{CONCLUSIONES}

En este artículo, se describen los resultados de un estudio que, a partir de la revisión teórica y de los resultados, analiza un conjunto de variables asociadas a la gestión escolar desde la perspectiva del profesorado que, se estima como hipótesis, tienen efecto sobre la calidad educativa. Por ejemplo, las dimensiones planificación, gestión de personas, recursos, procesos, satisfacción, resultados y el liderazgo.

Desde esta perspectiva, en el estudio encontramos efectos del liderazgo y de la gestión de personas sobre la satisfacción. En conjunto ambos factores expresan un $90,1 \%$ de la varianza. Por otro lado, la relación entre las variables satisfacción, liderazgo, recursos y procesos y los resultados, revela un $89,1 \%$ de la varianza. Finalmente los efectos de las variables liderazgo, recursos, procesos y resultados sobre la calidad educativa explican un $94,3 \%$ de la varianza.

Los resultados indican que en los centros educativos en que se percibe una gestión con un marcado acento en un liderazgo escolar, en un compromiso de los directivos para el desarrollo con el establecimiento y un reconocimiento de la labor docente, es valorada por los profesores como positiva en la percepción de la calidad educativa.

El modelo estudiado también entregó evidencia relacionada con las principales tendencias de los estudios sobre liderazgo escolar, las cuales modelan el efecto indirecto del liderazgo y otras variables asociadas a la gestión escolar como un encadenamiento de influencias que implica enfatizar objetivos académicos, llevar un seguimiento del desempeño de los profesores e incentivar el logro de los aprendizajes en los estudiantes.

Actualmente, más que nunca en nuestro país, se requiere rigurosidad y sistematicidad en todo el quehacer orientado a mejorar la calidad de la educación. Los resultados de este estudio sugieren profundizar en el conocimiento de las dinámicas de influencia e interacción entre sus principales 
involucrados: directivos docentes, profesores y estudiantes, ya que de acuerdo al modelo causal final (Fig.1) el liderazgo influye sobre la satisfacción y los resultados logrando, a través de esta última variable, afectar la calidad educativa.

Lo anterior confirma lo señalado por diversos estudios respecto de que el liderazgo que se ejerce en un centro educativo es, sin lugar a dudas, una de las variables más relevantes que influyen en el logro de los objetivos de la organización afectando, además, de manera indirecta, por medio de las interacciones que se producen en los diversos actores, el aprendizaje de los estudiantes e impactando así la calidad de la educación.

\section{REFERENCIAS BIBLIOGRAFICAS}

Albrecht, K., 1995

Hair, J., R. Anderson, R. Tatham, y W. Black, 2001

Cea, M.A., 2002

Coronel, J., 2008

Fundación Chile, 2003

Gaziel, H., M. Warnet \& I. Cantón, 2000

Jöreskog, K., D. Sörbom, 1993
Servicio al cliente interno. Editorial Paidós, Barcelona.

Análisis multiva riante. Editorial Printece Hall, Madrid.

Análisis multiva riable. Teoría y práctica en la investigación social. Editorial Síntesis, Madrid.

Liderazgo pedagógico: un reto y una posibilidad para la mejora educativa.

ICE. Universidad de Deusto, Bilbao, España. pp. 337-357.

Programa de certificación de la calidad de la gestión escolar. Santiago de Chile. p. 6-23.

La calidad en los centros docentes del siglo XXI. Propuestas y experiencias prácticas. Editorial La Muralla, Madrid. pp.51-193.

LISREL 8, Structural Equation Modeling with the Simple Language. Scientific Software International, Chicago. 
Lepeley, M. T., 2001

López Ruperez, F., 1997

Marchesi, Á. y

E. Martín, 1998

Maureira, O., 2008

MINEDUC, 2005

Ruiz Olabuénaga, J., 1995
Gestión y calidad en educación. Un modelo de evaluación. Editorial McGraw-Hill, Santiago de Chile.

La gestión de calidad en educación. Editorial La Muralla, Madrid.

Calidad de la enseñanza en los tiempos de cambio. Editorial Alianza, Madrid.

Avances del liderazgo directivo, actas del $V$ Congreso Internacional sobre Dirección de Centros Educativos. Universidad de Deusto, Bilbao. pp. 319-335.

Calidad en todas las escuelas y liceos. Sistema de aseguramiento de la calidad de la gestión escolar. MINEDUC, Santiago, Chile.

Sociología de las organizaciones. Universidad de Deusto, Bilbao. 